\title{
An Object-Level Feature Representation Model for the Multi-target Retrieval of Remote Sensing Images
}

\author{
Zhi Zeng* \\ Huizhou University, Computer Science \\ zengzhi@hzu.edu.cn \\ Zhenhong Du and Renyi Liu \\ Zhejiang University, Earth Science \\ duzhenhong_zju@163.com, liurenyi@mail.hz.zj.cn
}

\begin{abstract}
To address the problem of multi-target retrieval (MTR) of remote sensing images, this study proposes a new object-level feature representation model. The model provides an enhanced application image representation that improves the efficiency of MTR. Generating the model in our scheme includes processes, such as object-oriented image segmentation, feature parameter calculation, and symbolic image database construction. The proposed model uses the spatial representation method of the extended nine-direction lower-triangular (9DLT) matrix to combine spatial relationships among objects, and organizes the image features according to MPEG-7 standards. A similarity metric method is proposed that improves the precision of similarity retrieval. Our method provides a trade-off strategy that supports flexible matching on the target features, or the spatial relationship between the query target and the image database. We implement this retrieval framework on a dataset of remote sensing images. Experimental results show that the proposed model achieves competitive and high-retrieval precision.
\end{abstract}

Category: Smart and intelligent computing

Keywords: Remote sensing; Image processing; Spatial representation; 9DLT; Content-based remote sensing image retrieval

\section{INTRODUCTION}

Along with the rapid progress of satellite sensor technology and their application to high-resolution remote sensing images in Earth observation systems, a large amount of remote sensing data have become readily available for acquisition. In terms of spatial information, terrain geometry, and texture information, high-resolution remote sensing images have more features than middle or low-resolution images. To use the image database fully and to retrieve interesting information automatically and intelligently, a new efficient technology for multi-target retrieval (MTR) in an image, particularly in a specified region, is expected to be developed.

The number of image-processing applications for target retrieval is increasing, such as query by image content from IBM [1]. Most studies in this area have focused on content-based image retrieval (CBIR) and content-based

This is an Open Access article distributed under the terms of the Creative Commons Attribution Non-Commercial License (http://creativecommons.org/licenses/ by-nc/3.0/) which permits unrestricted non-commercial use, distribution, and reproduction in any medium, provided the original work is properly cited. 
remote sensing image retrieval (CBRSIR), and have achieved significant results. In these processes, the contents of an image, which specify several low-level features, such as color, texture, shape, longitude and latitude, and spatial relationships among objects, are the bases of multidimensional image feature vectors. Regarding the differences in imaging conditions of various forms of remote sensing images, we cannot exactly express image contents by using only a single feature. Therefore, constructing the comprehensive features of an image is the key to improving extraction performance [2]. However, if the combined features cannot be purified to form a unified model, then the accuracy of the similarity extraction and efficiency improvement of the images will be affected. For example, if we focus more on spatial relationship, then the detail of each target will be minimal. Thus, the efficiency of MTR will be higher than comparing the features of a single object. To effectively reveal the information retrieval process for remote sensing images, an objectlevel model is proposed, which can represent the contents of an image with overall accuracy. By using this model, we can retrieve and operate the information pre-stored in a symbolic image database (SID) with high efficiency, and neglect intrinsic information, such as color, texture, and shape. To date, research on feature representation models of image data for MTR remains limited. To build feature indices and to realize rapid retrieval, we propose an object-level feature representation model, based on a previous research on CBRSIR and the reference for MPEG-7 standards, starting with representing the contents of an image on an object-level feature, particularly the spatial relationship among targets.

The rest of this paper is organized as follows. Section II discusses related literature on representation techniques of image contents for CBIR or CBRSIR. Section III introduces calculation and representation feature values, and mainly describes the spatial representations of the extended nine-direction lower-triangular matrix (9DLT). Section IV presents a model of image content feature representation. Section V proposes an MTR model and similarity calculation. The last section presents several experiments to validate the accuracy of the content-based feature representation model and the efficiency of image target extraction. A conclusion to the study is also presented in this section.

\section{RELATED STUDIES}

In the past three decades, academia has achieved a large number of results on CBIR and CBRSIR. At present, CBIR has many successful applications in the fields of facial recognition, medical diagnosis, and trade registration. Most of these systems have adopted single feature or combined features as image indices [3-10]. CBRSIR is similar to CBIR, because both contain visual and geographic information. Several systems have focused on the issue of spectral retrieval, such as texture representation, and different combinations with spectral bands [11]. A special feedback approach has been employed to precisely describe the desired search characteristic in a remote sensing image [12]. Some researchers even presented a code stream of images for remote sensing image retrieval [13]. In addition, other scholars combined a scheme with an automatic classifier, and proposed the use of new feature 'texton histograms', to capture the weaktextured characteristic of remote sensing images for image retrieval [14]. Meanwhile, others applied a texture analysis approach, called the local binary pattern operator, to implement image retrieval [15]. Some of these studies even applied independent component analysis to extract independent components of feature values via linear combinations to realize multi-spectral image retrieval [16]; or adopted principal component analysis and a clustering technique to index remote sensing images for image retrieval [17]. Considering various features, such as color, texture, and spectra, a prototype model for CBRSIR based on color moment and gray level co-occurrence matrix feature was proposed [18]. A number of researchers combined several properties (color, texture, and points of interest) that were automatically extracted and immediately indexed images [19]. In addition, some researchers proposed a framework based on a domaindependent ontology to perform semantic retrieval in image archives [20]. Other scholars also presented a universal semantic data model for image retrieval [21]. Regardless of how a feature vector is established, this vector still depends upon the representation of contents in images. To date, the contents of images can be represented in numerous ways. Some approaches adopt a quad-tree structure or a quin-tree method that splits large-scale remote sensing images into sub-images, to extract multiple features, such as color and texture [22, 23]. Others use the 2D C-string to represent spatial knowledge of an image database [24]; or the spanning representation of an object to realize spatial inference and similarity retrieval in an image database, through directional relation referenced frames [25]. Others depict the relationships among spatial objects by using the methods of the nine-direction spanning area [26] or 9DLT [27]; and represent image colors by using pyramid technology [28]; or express an image by employing a symbol index, which is established in image space stratification [29]. All the aforementioned related representations include color, space, and subimages that belong to the feature representation method on image contents. Implementing rapid and accurate retrieval with a massive remote sensing image is difficult, because its features include various data types, resolution scales, and data sources. In our investigation, we analyze the contents of an image based on the MPEG-7 standard 
to organize the features of the image, build an SID, and index the SID to accelerate target retrieval.

\section{CALCULATING FEATURE VALUE}

The key to improving image retrieval efficiency is the index technique, which involves obtaining objects after image segmentation and building an SID for the image database. In the present study, we adopt a mature algorithm, called object-oriented multiscale image segmentation. The object-oriented image processing algorithm is a synthetic algorithm that fuses spectrum characteristics, geometric information, and structural information. This algorithm regards an object as a minimal process unit by retrieving its multiple characteristics to form a logical relationship among images and objects. Then, we analyze the image from the local to the entire level, and ultimately, implement its understanding. In general, multiscale image segmentation begins with any pixel by using a region merging method, from the bottom to the top, to form objects. Small objects can be merged to form large objects, and the size of each object must satisfy the demand of which the heterogeneity of a merged object is less than a given threshold. In this case, heterogeneity is decided by differences in the spectra and shapes of objects. However, various features correspond to different scales of observation, in which each feature can be extracted and accurately analyzed in an image layer on a proper scale [30]. In particular, we use the threshold value method on a multiple scale to segment an image.

After processing the calibration, segmentation, and raster vectorization of the image based on a specified region of latitude and longitude, the basic unit of the image is no longer a single pixel, but a polygon that is composed of homogeneous pixels. Each polygon can be used to calculate the spectral information of pixels, including shape, texture, color information, and topological relationships among the polygons. Next, we will introduce the method for calculating the feature vectors to implement the representation model.

\section{A. Shape}

Shape is a key feature used to differentiate two objects. It is also the basis for characteristic retrieval, and the classified process mentioned in the latter part of this paper. In general, in the field of object-level content retrieval, shape remains as the most basic feature for distinguishing objects. At present, two approaches are used to describe shapes: parametric and geometric approaches. In the present investigation, we adopt a geometric approach to characterize the shapes of different objects, namely, the model of centroid radii representation [31].

For an arbitrary polygon, such as the one shown in
Fig. 1(a), the results of resampling an image with the angle $\theta$ interval around, and counterclockwise to the $y$ axis, are shown as Fig. 1(b). Let $l_{k}$ be the distance between the centroid of the polygon, and the boundary sampling point. The shape descriptor of the polygon can be expressed by a centroid-radius model, as follows:

$$
\begin{aligned}
& F_{\text {shape }}=\left(l_{0}, l_{\theta}, l_{2 \theta}, \ldots, l_{i \theta}, \ldots, l_{(k-1) \theta}\right), \\
& k=\left\lfloor 360^{\circ} / \theta\right\rfloor, 0 \leq i \leq k-1 .
\end{aligned}
$$

The condition for measuring similarity between two polygons based on shape is: if and only if the numerical difference between the central radii in all directions is less than a given minor threshold value $\varepsilon$. That is, when two polygons are similar, the shape descriptor must satisfy the following regulation:

$$
\left|l 1_{i \theta}-l 2_{i d}\right|<\varepsilon, \forall i \in[0, k-1] .
$$

To ensure scale invariance of shape by using regulation (2), we need to normalize the Euclidean distance between the centroid and each vertex, within the range of $[0,1]$. In this study, we discuss most of the possible transformations between two feature vectors. One of these transformations involves the possible rotations between two shapes, and the distances that are independent from rotation, including the starting and ending points.

After transforming image shape into matrix space, we store data, using the antipole tree structure [32].

\section{B. Texture}

Different features in high-resolution images typically have similar spectral appearances to human vision. The mean value floating of a spectral feature may also cause similar spectra among different homogeneity samples to converge as similar modes in a feature space, thus resulting in spectra with similar features. This phenomenon is attributed to the human eyes being insensitive to some portion of visible light. Therefore, we can improve the reliability of retrieval results by using features, such as

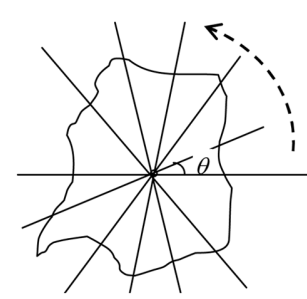

(a)

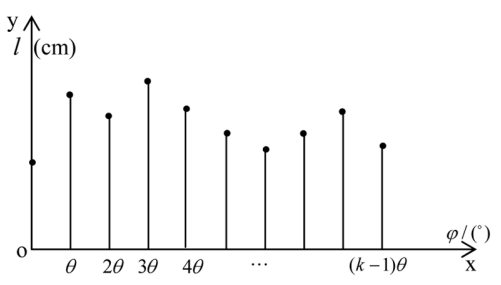

(b)
Fig. 1. Model of the centroid radii. (a) Resampled polygon with $\theta$ interval around, and counterclockwise to, the y axis; and (b) expression of the resampling result. 
shape, texture, and spatial relationships as references. Texture is a significant geometric (spatial) feature that can be used to distinguish among different objects and regions to reflect the changing discipline in gray space. A 2D Gabor filter is suitable for narrow-band coding of texture, because of its adjustable filtering direction, bandwidth, general band-center frequency, and optimal timedomain and spatial-domain analysis abilities. After finishing gray-scaling and normalization processes on image segmentation, we apply a Gabor filter to extract the texture feature of the objects. The Gabor filter function $g(x, y)$ is a $2 \mathrm{D}$ Gaussian function that is modulated by the complex sine window function $g(x, y)$. Its Fourier transform function $G(u, v)$ can be expressed by the following equations:

$$
\begin{gathered}
g(x, y)=\frac{1}{2 \pi \sigma_{x} \sigma_{y}} \exp \left[-\frac{1}{2}\left(\frac{x^{2}}{\sigma_{x}^{2}}+\frac{y^{2}}{\sigma_{y}^{2}}\right)+2 \pi i f x\right], \\
G(u, v)=\exp \left(-\frac{1}{2}\left[\frac{(u-f)^{2}}{\sigma_{u}^{2}}+\frac{v^{2}}{\sigma_{v}^{2}}\right]\right)
\end{gathered}
$$

where, $\sigma_{u}=\frac{1}{2 \pi \sigma_{x}}, \sigma_{v}=\frac{1}{2 \pi \sigma_{y}}$, and $\sigma_{x}, \sigma_{y}$ are the Gabor filter spatial range and bandwidth of the frequency domain, respectively. In this case, $(f, 0)$ is the central frequency in the filter of the orthogonal coordinate in the frequency domain. Let $g(x, y)$ be a function of the mother that generates the Gabor filter family. The set of functions $g_{m, n}(x, y)$, which is a complete non-orthogonal dataset, can be generated through rotation and scaling, according to Eq. (4).

$$
g_{m, n}(x, y)=a^{-2 m} g\left(x^{\prime}, y^{\prime}\right)
$$

where $x^{\prime}=a^{-m}\left(x \cos \theta_{n}+y \sin \theta_{n}\right), y^{\prime}=a^{-m}\left(-x \sin \theta_{n}+y \cos \theta_{n}\right)$, a $>1, \theta_{n}=n \pi / K, m=0,1, \ldots, \mathrm{S}-1$, and $n=0,1, \ldots, K-1$. Parameter $\theta_{n}$ is the counterclockwise rotation angle along the filter axis. $S, K$ are the total scale and rotation, respectively. After obtaining the energy value of each filter and the convolution of the image, we calculate the mean value and the mean square deviation of the filtering value on the energy of each object. Finally, we mark the texture feature vector of the object, as shown in Eq. (5).

$$
\begin{aligned}
F_{\text {texture }}= & \left\{\mu_{0,0}^{\text {texture }}, \sigma_{0,0}^{\text {texture }}, \mu_{0,1}^{\text {texture }}, \sigma_{0,1}^{\text {texture }}, \ldots,\right. \\
& \left.\mu_{k, l}^{\text {texture }}, \sigma_{k, l}^{\text {texture }}, \ldots, \mu_{K-1, L-1}^{\text {texture }}, \sigma_{K-1, L-1}^{\text {texture }}\right\} .
\end{aligned}
$$

where, $K$ is the central frequency, and $L$ is the directional angle. $k=0,1 \ldots, K-1, l=0,1, \ldots \ldots, L-1$, and $E_{k, l}(x, y)$ is the filtering energy value of the filter $(k, l)$. Normalization is required to proceed toward $E_{k, l}(x, y)$, to ensure that the energy value of each element in the energy information is not affected by the actual size. We commonly use $E_{k, l}(x, y)=\sum p(x, y)^{2}$ to calculate the energy value, according to the gray value $p(x, y)$ in loca- tion $(x, y)$. Finally, the mean value $\mu$ of the energy, and the mean square deviation $\sigma$ of the target object $(n \times n$ pixels), can be obtained as Eqs. (6) and (7), respectively.

$$
\begin{gathered}
\mu_{k, l}^{\text {texture }}=\frac{\sum_{x} \sum_{y} E_{k, l}(x, y)}{n \times n}, \\
\sigma_{k, l}^{\text {texture }}=\sqrt{\frac{\sum_{x} \sum_{y}\left(E_{k, l}(x, y)-\mu_{k, l}^{\text {texture }}\right)^{2}}{n \times n} .}
\end{gathered}
$$

\section{Spatial Representation of the Extended 9DLT}

The spatial representation of an image describes the spatial relationships among objects to easily distinguish images with multiple targets. The spatial relationships in an image can be classified into two categories: positional and directional relationships. The former can be represented by a $2 \mathrm{D}$ string; whereas, the latter can be represented by 9DLT methods [25]. For a calibrated remote sensing image within the region of a certain latitude and longitude, the directional relationship relative to the four corners among the objects is confirmed. In this section, we introduce problem definitions and preliminary concepts, through formal methods.

DEFINITION 1. Let $\alpha=\left(\alpha_{1}, \alpha_{2}, \ldots, \alpha_{k}\right)$ be a set of objects in the same image. Hence, $\alpha_{i}$ is a subset of $\alpha$.

DEFINITION 2. The spatial relationship between two objects can be defined as one of the codes in nine directions, which is called 9DLT.

DEFINITION 3 (The 9DLT matrix). Let $V=\left\{v_{1}, v_{2}, v_{3}\right.$, ..., $\left.v_{m}\right\}$ be composed of $m$ distinct sets of objects, and $Z$ be composed of $z_{1}, z_{2}, z_{3}, \ldots, z_{s}$ in order, where $\forall i=1,2, \ldots$, $s, z_{i} \in V$. Suppose $C$ is a collection of 9D encodings, as shown in Fig. 2(a). Each direction code can then be used to specify the spatial relationship between two objects.

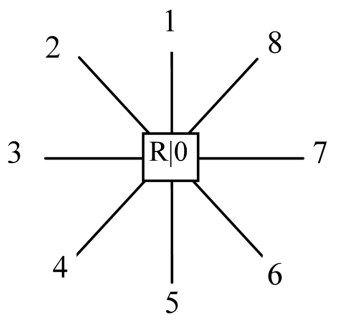

(a)

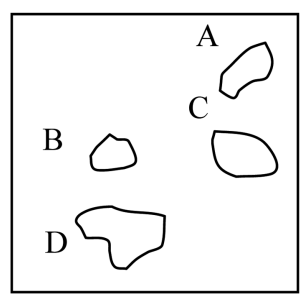

(b)
Fig. 2. Representation of nine-direction lower-triangular. (a) Nine-direction code and (b) symbolic figure of the object. 


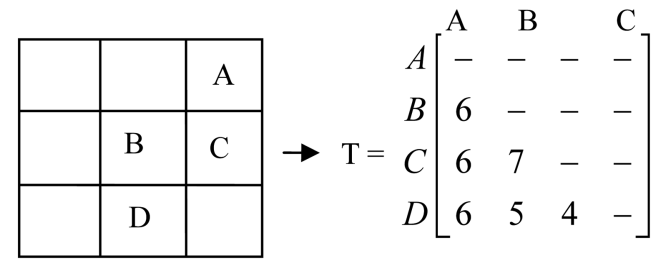

(a)

(b)

Fig. 3. Map of the matrix expression in nine-direction lowertriangular (9DLT). (a) Direction map in grids between objects and (b) matrix expression of four objects in 9DLT.

Thus, a 9DLT matrix $T$ is an $s \times s$ matrix that is composed of $t_{i j}$, which belongs to the collection of 9D encoding $C$. The item $t_{i j}$ at row $i$ column $j$ represents the direction code from $z_{j}$ to $z_{i}$, only when $i$ and $j$ satisfy the condition $j<i \in(1, s)$.

As shown in Fig. 2(a), let $R$ be the referred object expressed by 0 , in which we define the direction code in a $45^{\circ}$ interval from the northern counterclockwise, as $1 \ldots 8$. Each object from the source image will be represented by one centroid in a 9DLT expression. Fig. 2(b) shows a feature image that contains four objects. Fig. 3(a) exhibits the direction map in the grid between the objects; whereas, the direction code of the LT matrix in Fig. 3(b) demonstrates the spatial relationships among objects. The 9DLT string is $(A, B, C, D, 6,6,6,7,5,4)$ in column order. A relationship between two objects exists in the matrix.

DEFINITION 4. A pattern consists of the sets of objects and the spatial relationships among these objects. For example, $\alpha=\left(\alpha_{1}, \alpha_{2}, \ldots, \alpha_{k}, \alpha r_{1}, \alpha r_{2}, \ldots, \alpha r_{m}\right)$ is a pattern, $\alpha_{i}$ is an object and $\alpha r_{j}$ is the corresponding spatial relationship, where $1 \leq i \leq k, m=C_{2}^{k}=k(k-1) / 2,1 \leq j \leq m$, and $k \geq 2$. That is, the spatial relationships between any two objects in this pattern are recorded. The length of a pattern is equal to the amount of objects. A pattern with a length that is equal to $k$ is called the $k$-pattern.

\section{Constraints:}

(1) An item or object in a pattern is stored in alphabetical order.

(2) No spatial relationship exists, if the length of a pattern is equal to 1 .

The 9DLT expression is in accordance with the definition of the pattern.

DEFINITION 5. Pattern $\alpha=\left(\alpha_{1}, \alpha_{2}, \ldots, \alpha_{i}, \alpha r_{1}, \alpha r_{2}, \ldots\right.$, $\left.\alpha r_{m}\right)$ is a sub-pattern of pattern $\beta=\left(\beta_{1}, \beta_{2}, \ldots, \beta_{j}, \beta r_{1}, \beta r_{2}\right.$, $\left.\ldots, \beta r_{n}\right)$, where $\left(\alpha_{1}, \alpha_{2}, \ldots, \alpha_{i}\right)$ is a subset of $\left(\beta_{1}, \beta_{2}, \ldots, \beta_{j}\right)$, where $j \geq i \geq 2$. The spatial relationship between any two items in $\alpha$ is the same as in $\beta$. Pattern $\beta$ contains pattern $\alpha$, where $\beta \supseteq \alpha$. The amount of a sub-pattern is $N=$ $C_{i}^{i-1}+C_{i}^{i-2}+\cdots+C_{i}^{2}$. For example, pattern $\alpha=(A, B, C, 6$,
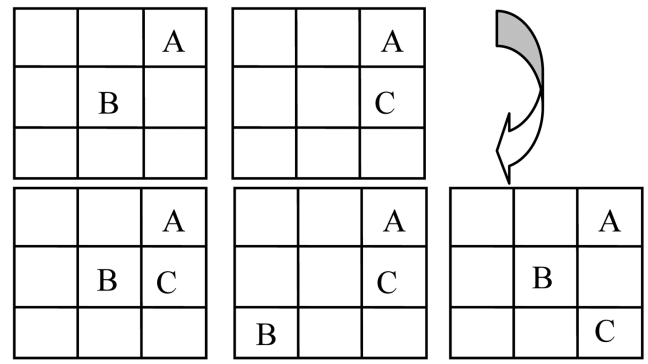

Fig. 4. Generation of candidate 3-pattern from 2-pattern.

$6,7)$ is a sub-pattern of pattern $\beta=(\underline{A}, \underline{B}, \underline{C}, D, \underline{6}, \underline{6}, 6, \underline{7}$, $5,4)$, because $(A, B, C)$ is a subset of $(A, B, C, D)$, and the code values of the spatial relationship of objects $A, B$, and $C$ are the same as the code values underlined in pattern $\beta$.

DEFINITION 6. The minimum support is the amount of objects that satisfy the spatial relationships, which is equal to the required amount of search objects.

Inference 1. Two $k$-patterns can be joined, only if $k-1$ objects and the corresponding relationships between them are the same, and $k$ satisfies condition $k \geq 2$.

Inference 2. Suppose a pattern does not contain any $(k-1)$ pattern; then, this pattern cannot be contained in the $k$-pattern.

Inference 3. The pattern of feature images and their specific sub-pattern can be obtained from a 9DLT string. By contrast, if ( $k-1)$-pattern, $k-1$ objects, and the spatial relationships in the object sets are given, then the relative candidate sets of the $k$-pattern can be acquired.

Generating candidate sets can significantly help object retrieval. To extract the image with the object $(A, B, C)$ of minimum support 3 and spatial relationship $\alpha r$ in the image database, two 2-patterns are required, namely, ( $A$, $B, 4)$ and $(A, B, 5)$, in which the same object $A$ belongs to both patterns, and satisfies the joining condition. Then, we can calculate the candidate 3-pattern $(A, B, C, 4,5, \Delta)$. As shown in Fig. 4, the possible results are $(A, B, C, 4,5$, $7),(A, B, C, 4,5,8)$, and $(A, B, C, 4,5,6)$. The direction codes of the possible relationship between $B$ and $C$ are 7, 8 , and 6; therefore, the spatial representation model is $A B C(4,5, X:\{7,8,6\})$.

Similarly, the 9DLT string of each image is known in the image database. That is, the spatial relationship between objects has been confirmed, and the problem of finding all images that satisfy minimum support is the process of matching patterns. In fact, the process can be converted to search the LT matrix with a problem on inclusion relationship. As shown in Fig. 5, according to the difference of the given objects and the minimum support, the position of matching matrix $P$ in the LT matrix may only be a part of the relation direction codes. The range of mapping to candidate matrix $C$ is also in $k \times k$. 
The description of the match algorithm is as follows.

Algorithm 1 Match algorithm
Input: $k$-pattern of a 9DLT string that prepares to
match the object $O_{i}(2 \leq i \leq k)$ and the minimum
support, image feature library.
Output: Image ID
1: begin
2: Convert $k$-pattern into matrix $P$ in an object-
3: labeled order;
4: $\quad$ Make $S$ generate the candidate matrix $C_{i}$
5: $\quad$ in the object-labeled order.
6: $\quad$ If $\left.P \subseteq C_{i}\right)$
7: $\quad$ Search the image ID by using the
8: $\quad$ Output the image ID;
9: end $\quad$

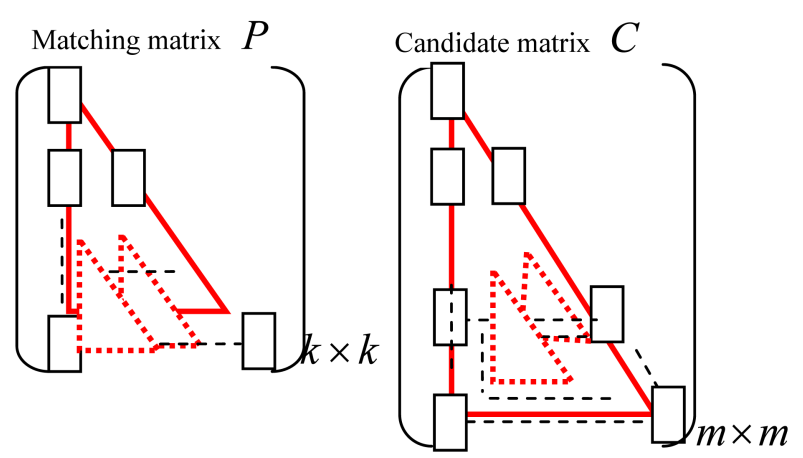

Fig. 5. Map of the candidate matrix to match the threshold.

\section{THE OBJECT-LEVEL FEATURE REPRESEN- TATION MODEL}

Typically, a data model is a framework that can be used to provide representation for information, and an operation method in the database system. The object-level feature representation model belongs to a section of this data model. For remote sensing images, the data model also includes metadata, such as location, resolution, and light intensity. However, the standard of measurement for a content retrieval system determines the efficiency and accuracy of extraction. Hence, each image needs a good model with an efficient content-based representation. Moreover, selecting a formula for similarity calculation is

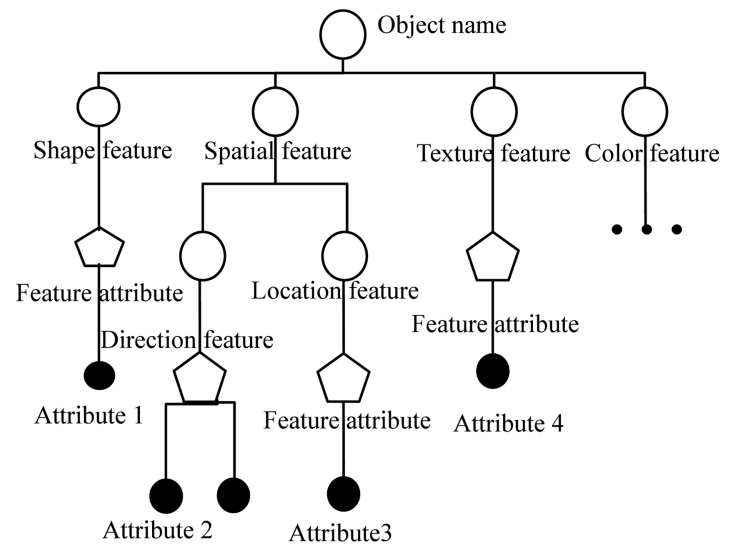

Fig. 6. Model of representation of feature objects.

also vital. Based on this concept, we present the objectlevel feature representation model for the image data in the next section.

According to MPEG-7 standards and the object-oriented concept, the object-level feature representation model for image data is described as a structural tree via layers [33]. As Fig. 6 shows, the first layer is the object name, while the second layer is the feature name of the feature information that the object contains. Further down are the layers for sub-features, feature attributes, attribute values, etc. Constructing this structural tree is convenient for indexing feature information.

The overall model of the feature image can be represented by a formal method, as follows:

$$
\begin{gathered}
\text { Feature Im age }=(\operatorname{Im} \text { ageID, MataData, Objs }), \\
\text { MetaData }=(\text { Location, Scal, Im ageID }), \\
\text { Objs }=\left\{\text { obj }_{1}, o b j_{2}, \ldots, o b j_{n}\right\}, \\
O b j_{i}=\left(o b j I D, o b j N a m e, \ldots, F_{o b j}\right), 2 \leq i \leq n . \\
F_{o b j}=(o b j I D, V, E A, M A),
\end{gathered}
$$

where, $E A$ stands for the description of the object extraction algorithm, and $M A$ stands for the description of the object matching algorithm.

$$
V=\left\{F_{\text {shape }}, F_{\text {space }}, F_{\text {texture }}, \ldots\right\} .
$$

We adopt the centroid-radii model $F_{\text {shape }}=($ objID, Centriod, Radii $)=$ in Section III-A, the 9DLT extended model $F_{\text {space }}=\left(\right.$ objID $\left., F_{\text {local }}, F_{\text {direc }}\right)$ in Section III-C, and the calculation value of the different direction angle energy $F_{\text {texture }}$ in Section III-B. We choose parameters that refer to the methods in the literature [34]. The color feature of an object can be expressed as $F_{\text {color }}=\left\{\mu_{\text {color }}, \sigma_{\text {color }}\right\}$, through the mean value and the mean square deviation of its color. 
Through this model, we can express the content of multiple targets in an image by using multiple records to represent a single object, such as color, shape, and texture features. Then, a logical expression is implemented by the spatial relationship among objects. Thus, we transform the MTR problem into a record-querying problem to enable the image indexing technology to further accelerate target retrieval in CBRSIR.

\section{MTR AND THE SIMILARITY METRIC}

MTR is a branch of CBRSIR, which is an application based on multiple targets, and the representation of spatial relationships. In general, users employ two forms for CBIR, namely, sample and sketch retrievals. In terms of searching image-by-image, or ranking by similarity value, the key point is similarity calculation of the model of feature representation as a metric standard. In our investigation, the image retrieval model uses a sketch retrieval interface (Fig. 7). A feature-matching model with the prepared conditions can be generated from the editing interface, as well as from the specified shape, texture, and spatial relationships of the targets, within a certain resolution and region in the sketch.

The system has stored the information of each sample object that contains their shape, texture, and spectral features in a specified resolution in SID; hence, the system can label each selected target in alphabetical order (from left to right, and row-by-row). The system can also use low-level features and the location of the object centroid to generate the feature representation model of the preextracted image.

For a multiple-target case, the spatial similarity on shape among the targets is between two feature images with a specified resolution. We can line the centroid of the polygon with its target to obtain the shape parameter according to Eq. (8).

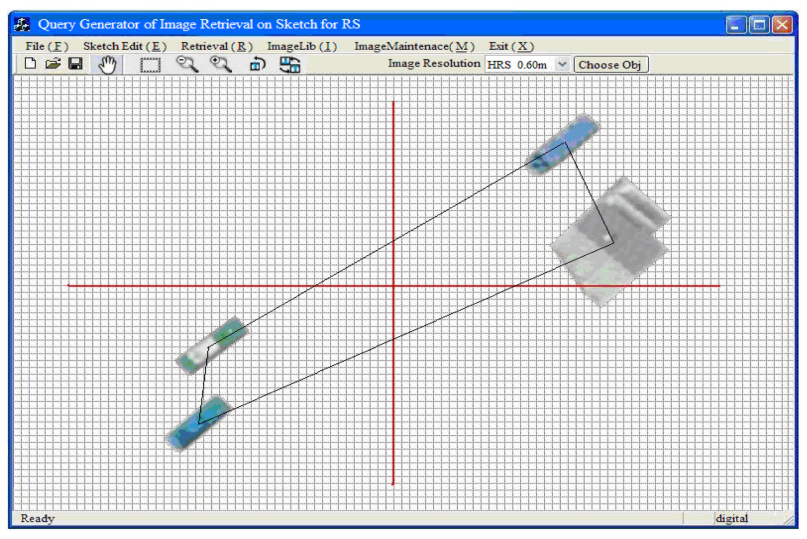

Fig. 7. Paint canvas of drawing image for the testing system.

$$
F=\|C\|^{2} / 4 \pi S,
$$

where, $C$ is the perimeter of the region and $S$ is the area of the region. The shape parameter $F$ reflects the compactness of the region to a certain extent, in which the region is dimensionless and insensitive to the change in scale and rotation. It also does not have a fixed range. In general, the greater the value is, the less compact and more regular the shape is. The difference between Eq. (9) and Eq. (1) is that the polygon shape in the latter is more complex than that in the former. When precision is considered, the shape, which is formed by multiple targets, is more regular.

After normalizing the object of the target, the value of each item in the texture feature vector, which is extracted by Gabor filtering, is within the range of $[0,1]$. When calculating similarity distance, we endow each item with the same weight coefficient. The total similarity of a single object regarding texture feature can be calculated by using Eq. (9) [35].

$$
S_{i}=\frac{\omega_{1} a_{1}+\omega_{2} a_{2}+\cdots+\omega_{n} a_{n}}{\omega_{1}+\omega_{2}+\cdots \omega_{n}}, 1 \leq i \leq k,
$$

where, $k$ is the number of target objects, and $\omega_{n}$ and $a_{n}$ stand for the weight coefficient and the similarity of the number $n$ feature vector in all directions, respectively.

We commonly used 9DLT string to represent the location of the centroid of objects within an angle of $45^{\circ}$ during MTR, according to point group distribution similarity calculation [36]. The correlative parameters include spatial direction and distance range. Then, the spatial similarity can be calculated by Eq. (10).

$$
S_{\text {space }}=\sqrt[2]{\text { Sim_diret } \times \text { Sim_dist }},
$$

where, Sim_diret $=\left|\cos \theta_{1}-\cos \theta_{2}\right|, 0 \leq \theta_{1}, \theta_{2} \leq \pi / 4$, Sim_dist $=1-\frac{\left|r_{1}-r_{2}\right|}{\max \left(r_{1}, r_{2}\right)}$, and $r_{i}=\sqrt{x_{i}^{2}+y_{i}^{2}}, i \in\{1,2\}$.

$\theta_{i},\left(x_{i}, y_{i}\right)$, and $i \in\{1,2\}$ stand for the angle between the query object, the object in the image library in eight directional axes of the Cartesian coordinate system, and the coordinate of the object, respectively. The importance of the feature values on MTR varies during target matching. The experiment empirically provides different weight coefficients to calculate the total similarity of the image. Thus, the similarity distance on the feature vector between the querying sketch and the images from a library is defined as Eq. (11).

$$
D=\sum_{i \in L} \omega_{i} \times\left(\frac{F_{i}-F_{i}^{\prime}}{\left|F_{i}\right|+\left|F_{i}\right|}\right)^{2},
$$

where, $i$ is the feature dimension, $\omega_{i}$ is the weight coefficient of feature $F_{i} \in V$, and $V=\left\{F_{\text {shape }}, F_{\text {space }}, F_{\text {texture }}, \ldots\right\}$. 


\section{EXPERIMENTS AND CONCLUSION}

\section{A. Testing and Experiments}

We collect 3,650 pieces of high-resolution images from different sensors as samples with the amount reaching the terabyte level. Each image measures $0.60 \mathrm{~m}$, and has a resolution of 5,000 $\times 5,000$. We use the segmentation algorithm of a multiscale threshold value based on the minimum of heterogeneity with a scale parameter of 150 and a color parameter of 0.90 to conduct a quantitative analysis on the object-level feature representation model to validate its rationality and retrieval efficiency.

Our testing system adopts $\mathrm{C}++$ language, ArcGIS, and MATLAB to implement the image retrieval system based on the content of the image, in which high-resolution images processed by geometric calibration are covered. After object-oriented segmentation obtains and vectorizes the target objects, the system calculates the feature values of shape and texture, and imports them into the image library, along with the image metadata, thus forming an SID from the feature model. Then, we calculate the similarity, and match it with the query vector feature generated from the retrieval sketch by sample querying in the SID to obtain the result sets. The specific retrieval process is illustrated in Fig. 8.

Fig. 9(a) is a QuickBird $0.6 \mathrm{~m}$ high-resolution image before segmentation, which contains extraction targets. Fig. 9(b) is the result of the symbolic image after image segmentation was performed with the given parameters to achieve minimum heterogeneity.

For the symbolic image, raster vectorization is performed after image segmentation. Then, to obtain the relative coordinate of the centroid of the object, a coordinate in the left corner, within the given longitude and latitude, is designated as a relative coordinate. Next, we progressively scan the centroid coordinate of the polygon from each object from left to right, and label them in alphabetical order. For convenient calculation, we use the poly- gons marked A to D, as shown in Fig. 9(b). The steps for the entire process are described below.

First, for the representation model of the centroid-radii, we use the antipole tree structure, which is described in Section III-A to store the data. Then, we proceed to gray scaling and normalization of the object resulting from the segmentation by using the calculation model of texture in Section III-B. Normalization, which calculates the energy ratio between each element and the maximum ratio of size and direction of the energy sets of the image target pixel, can eliminate the influence of the actual size of objects. Thus, we obtain the filtering energy values of the Gabor filters under different frequencies and azimuth angles by calculating the mean value and the mean square deviation of the energy values of each object, and by considering them as the texture feature vector of the object. This study applies the result from the Fourier transform to the convolution translation between a group of different frequencies and Gabor filters with different directions

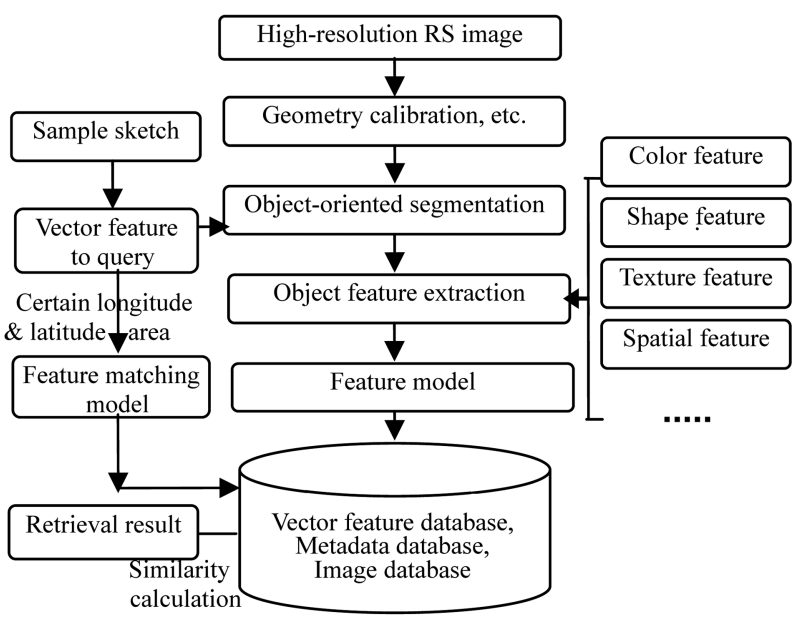

Fig. 8. Retrieval process of the testing system.

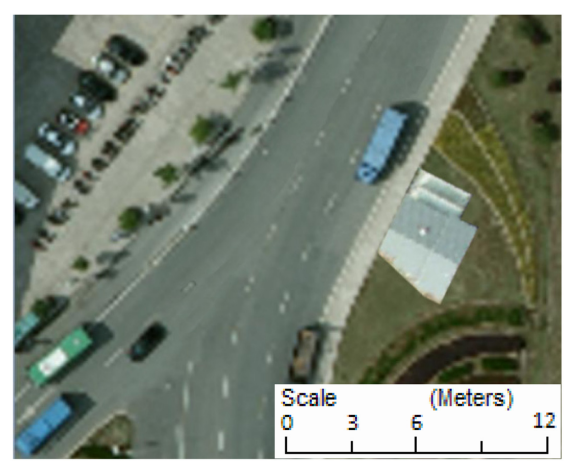

(a)

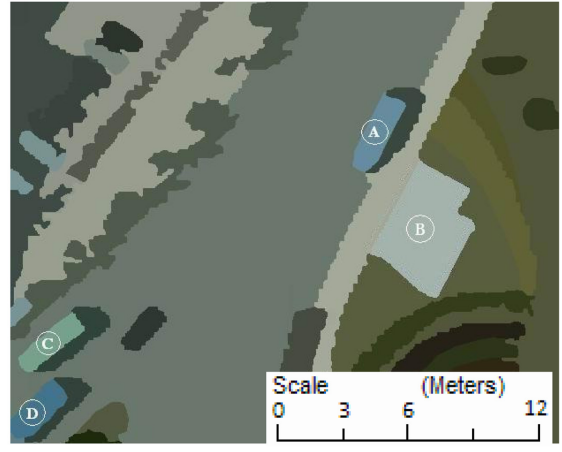

(b)

Fig. 9. Images before and after object-level segmentation. (a) Original image of high-resolution remote sensing and (b) symbolic image using object-level segmentation. 


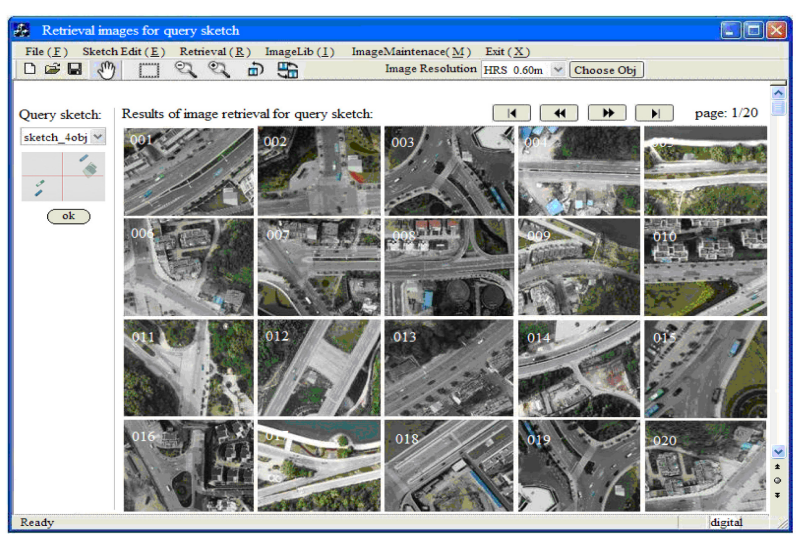

Fig. 10. Results of image retrieval by query sketch.

and objects [37]. In this case, we only calculate the feature values by using a Gabor filter to construct a texture feature vector.

Afterward, we obtain 9DLT string $(A, B, C, D, 5,4,4$, $4,4,4)$, according to Section III-C.

Finally, Section III explains that the system can organize all relevant calculation results as records in the model library. According to the query generator of the image retrieval on the sketch, our testing system can calculate the similarity among objects, which is conveniently derived from an image library and sketched. Fig. 10 displays the top 20 results of image retrieval by query sketch. All images are ranked according to the trade-off strategy on similarity, which includes features and spatial relationships between objects.

\section{B. Analyzing the Experimental Results}

The values of the weight coefficient, which are based on the error source from the correlation, and the similarity of each feature vector in the image parsing process and in the multi-experiment result, are shown in Table 1, where the spatial feature specifies the shape among objects. Meanwhile, Section V presents the combined metric of spatial similarity on shape among targets by using Eq. (9), in which a trade-off strategy to support flexible matching policy and to calculate similarity distance was applied by tuning the ratio between spatial similarity and feature vector.

If the distance between different tolerance feature vectors is short, then the similarity is great. For the query results of the image, we take the top 200 images to ana-

Table 1. Weight coefficient at each dimensional feature vector

\begin{tabular}{ccccc}
\hline $\begin{array}{c}\text { Weight } \\
\text { coefficient }\end{array}$ & $\begin{array}{c}\text { Object shape } \\
\text { feature }\end{array}$ & $\begin{array}{c}\text { Texture } \\
\text { feature }\end{array}$ & $\begin{array}{c}\text { Color } \\
\text { feature }\end{array}$ & $\begin{array}{c}\text { Spatial } \\
\text { feature }\end{array}$ \\
\hline$\omega$ & 0.3 & 0.3 & 0.2 & 0.2 \\
\hline
\end{tabular}

Table 2. Testing result of recall and precision for different targets

\begin{tabular}{|c|c|c|c|c|c|c|c|c|}
\hline & \multicolumn{4}{|c|}{ Recall } & \multicolumn{4}{|c|}{ Precision } \\
\hline & \multicolumn{4}{|c|}{ Number of target } & \multicolumn{4}{|c|}{ Number of target } \\
\hline & 1 & 2 & 3 & 4 & 1 & 2 & 3 & 4 \\
\hline Test 1 & 0.62 & 0.59 & 0.57 & 0.52 & 0.48 & 0.36 & 0.30 & 0.26 \\
\hline Test 2 & 0.65 & 0.58 & 0.57 & 0.42 & 0.46 & 0.35 & 0.32 & 0.31 \\
\hline Test 3 & 0.56 & 0.55 & 0.52 & 0.37 & 0.42 & 0.37 & 0.35 & 0.32 \\
\hline Test 4 & 0.57 & 0.51 & 0.50 & 0.45 & 0.49 & 0.42 & 0.40 & 0.31 \\
\hline Test 5 & 0.68 & 0.64 & 0.52 & 0.48 & 0.41 & 0.36 & 0.32 & 0.29 \\
\hline Test 6 & 0.75 & 0.72 & 0.67 & 0.62 & 0.52 & 0.51 & 0.39 & 0.37 \\
\hline Test 7 & 0.74 & 0.67 & 0.63 & 0.57 & 0.43 & 0.31 & 0.27 & 0.26 \\
\hline Test 8 & 0.63 & 0.57 & 0.49 & 0.47 & 0.38 & 0.30 & 0.27 & 0.23 \\
\hline Test 9 & 0.72 & 0.68 & 0.65 & 0.58 & 0.38 & 0.32 & 0.30 & 0.24 \\
\hline Test 10 & 0.68 & 0.67 & 0.64 & 0.60 & 0.44 & 0.34 & 0.29 & 0.27 \\
\hline Avg. & 0.66 & 0.62 & 0.58 & 0.51 & 0.44 & 0.36 & 0.32 & 0.28 \\
\hline
\end{tabular}

lyze the retrieval accuracy of the system. The equations for normalized precision and normalized recall are as follows [38]:

$$
\begin{aligned}
& P_{\text {norm }}=1-\frac{\sum_{i=1}^{R}\left(\log \text { rank }_{i}-\log i\right)}{\log (N ! /(N-R) ! R !)}, \\
& R_{\text {norm }}=1-\frac{\sum_{i=1}^{R}\left(\text { rank }_{i}-i\right)}{(N-R) R} .
\end{aligned}
$$

where, $N$ is the total number of images in the image library, $R$ is the number of images extracted, and $i$ is the position of the extracted images. rank $k_{i}$ is the actual ranking of the retrieved images.

Table 2 shows the typical 10 times testing result on the recall/precision from the image library, when we extract a different number of targets. Table 2 also indicates that when the search targets increase, the target itself, and the complexity of the spatial relationship among objects, also increase, thus resulting in a decline in the values of recall and precision. A reverse interdependence relationship occurs between recall and precision. Thus, if we raise the recall of the output, then the precision will be reduced; and vice versa. In fact, the number of images that can be extracted will increase, if the tolerance value of similarity increases; while the distance of the feature vector $D$ is correspondingly reduced. In addition, both recall and precision ratios will exhibit a slight increase. Fig. 11(a) shows that, with the increase of search target numbers, the average normalized recall/precision presents a certain decline. An inverse relationship exists between normalized recall and precision. Fig. 11(b) is a comparison graph of recall and precision ratios, which were obtained 


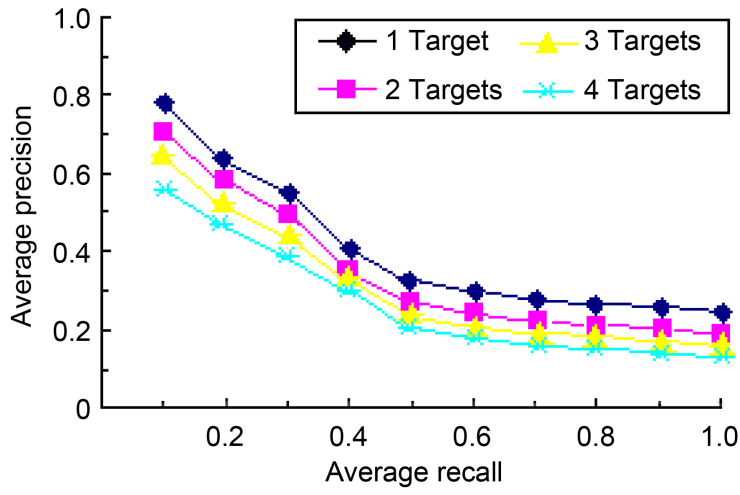

(a)

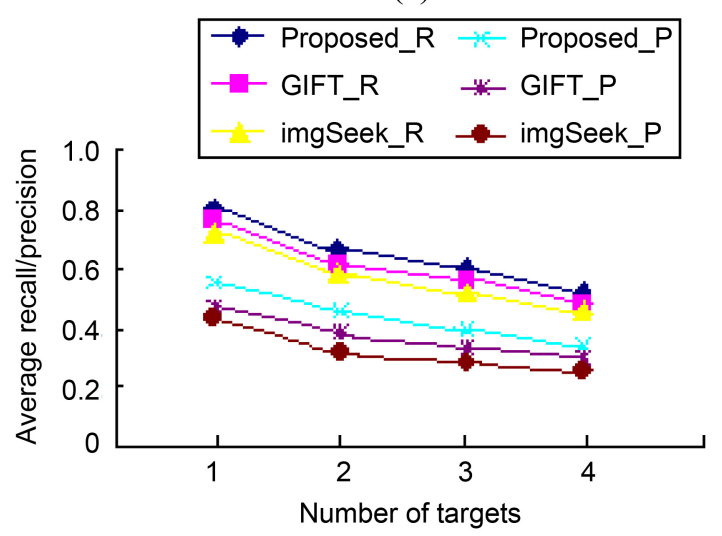

(b)

Fig. 11. Case of average recall and precision with targets' number. (a) Relationship of average recall and average precision with different targets. (b) Comparison of average recall and precision with targets for different algorithms.

by using different methods to retrieve the number of targets. The graph shows that the method we proposed is more effective than the two methods introduced in the literature [6]. The broken lines in Fig. 12 indicate the slight increase in recall and precision, when the tolerance values of similarity increase from $1 \%$ to $10 \%$ by applying the same method.

Next, we discuss the change in time-consuming retrieval from the perspective of the retrieval efficiency of a system, thus combining the number of targets that require retrieval under the amount of different image data. Here, we ignore the majority of the time on image calibration and the generation of a vector feature library. The total time of the image retrieval process is shown as follows:

$$
\begin{aligned}
T_{\text {total }}= & T_{\text {gen_cond }}\left(N_{\text {objects }}\right)+T_{\text {search }}\left(N_{\text {imgss }}, \text { Area }(\text { lon }, \text { lat })\right) \\
& +\sum_{i=1}^{n} T_{\text {trans }}\left(N_{\text {data }}(i)\right) .
\end{aligned}
$$

where, $T_{\text {gen_cond }}\left(N_{\text {objects }}\right)$ stands for the time of generation from the sketch, which is relative to the amount of search

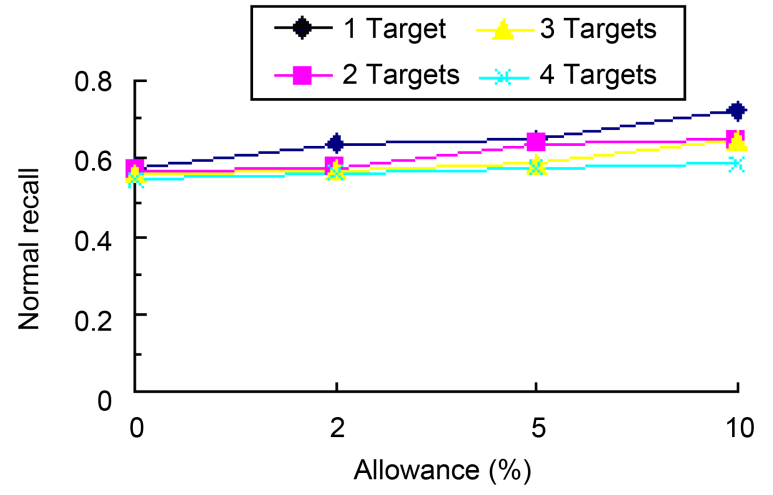

(a)

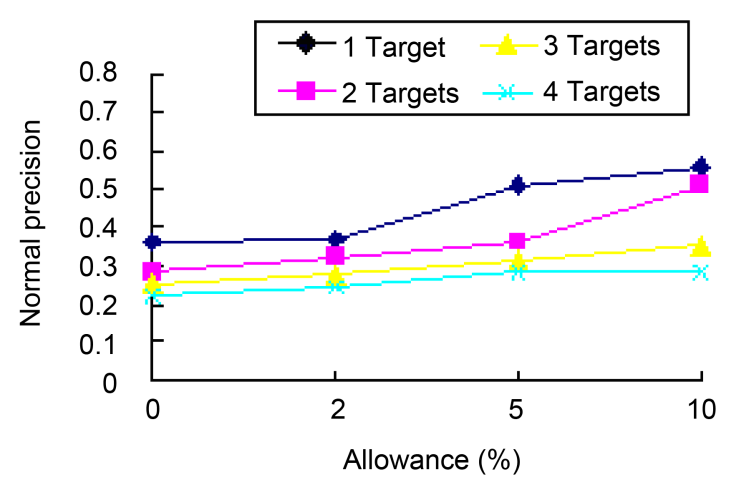

(b)

Fig. 12. Case of normalized recall and precision with targets' number. (a) Comparison of normalized recall with different allowance values. (b) Comparison of normalized precision with different allowance value.

targets $\left(N_{\text {objects }}\right) \cdot T_{\text {search }}\left(N_{\text {imgs }}\right.$, Area $($ lon, lat $\left.)\right)$ is relative to the amount of images, and the region of a given longitude and latitude, which represents the search time of the model that matches that from SID. $T_{\text {trans }}\left(N_{\text {data }}(i)\right)$ is the transportation and display time of $n$ images that satisfy the given threshold value, and is relative to the amount of images $N_{\text {data }}(i)$. Fig. 13(a) shows a time-consuming situation under the same condition, which includes the number of SID for searching different targets to constrain the display time of the top 20 miniature images that satisfy the given threshold value of similarity. Based on the chart, spatial complexity increases with the increase in the number of search targets, in which the search time also presents an upward trend. Fig. 13(b) shows the average time consumed by querying different targets in quantity among the methods proposed in this paper, GIFT, and imgSeek, under the same image library and query conditions. Although the total consumed time in our method is slightly more than that of the GIFT method, its recall and precision are higher than those of the other two methods. The results are consistent with the previous analysis shown in Fig. 11(b). In summary, the experiments dem- 


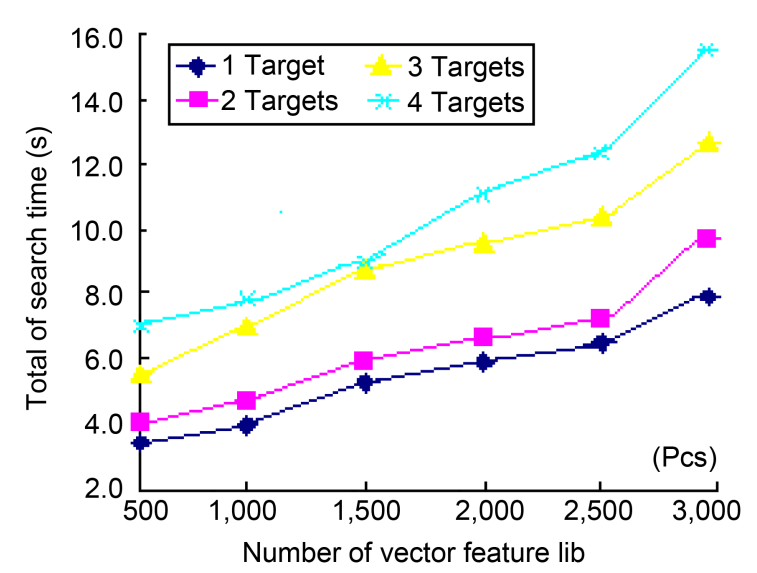

(a)

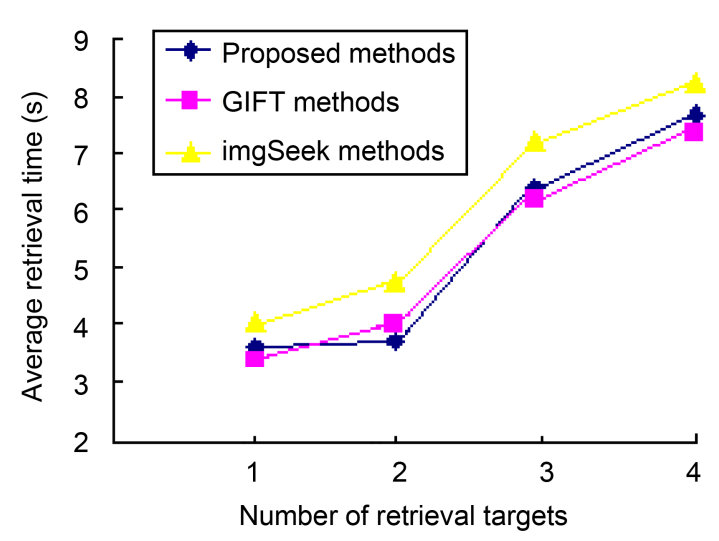

(b)

Fig. 13. Time costs for number of feature lib with number of retrieval targets. (a) Trends of total of search time and number of vector feature lib with different targets. (b) Comparison of average retrieval time and number of retrieval targets under different algorithms.

onstrate the effectiveness of our proposed framework, which has better precision than the other methods, particularly in multiple targets. However, our method requires more computing power.

\section{Conclusion}

This study, which was based on the inherent characteristics of high-resolution images, such as shape, texture, color, and spatial features, proposed an object-level feature representation model for image contents. Through experiments, we verified the feasibility and the effectiveness of the proposed model with two quantitative indicators in recall and precision. Given the difference among amount, classification, and spatial complexity of features among images, the proportion of the overall shape of two images also results in differences in recall and precision in spatial relationships with a fixed resolution or scale.
Therefore, the time spent on searching for targets is different, and the performance is also affected. Further studies on evaluation standards of the similarity metric, particularly for multiple targets, should be conducted to improve the efficiency and precision of retrieving targets.

\section{AKNOWLEDGMENTS}

This work is supported in part by the National Natural Science Foundation of China (No. 41001227) and the doctoral startup fund of Huizhou University (No. C513.0201). We would also like to thank the referees for their valuable comments and suggestions.

\section{REFERENCES}

1. M. Flickner, H. Sawhney, W. Niblack, J. Ashley, Q. Huang, B. Dom, ... P. Yanker, "Query by image and video content: the QBIC system," Computer, vol. 28, no. 9, pp. 23-32, 1995.

2. L. Z. Lu, R. Y. Ren, and N. Liu, "Remote sensing image retrieval using color and texture fused features," China Journal of Image and Graphics, vol. 9, no. 3, pp. 74-78, 2004.

3. E. Guldogan and M. Gabbouj, "Feature selection for content-based image retrieval," Signal, Image and Video Processing, vol. 2, no. 3, pp. 241-250, 2008.

4. R. Kapela, P. Sniatala, and A. Rybarczyk, "Real-time visual content description system based on MPEG-7 descriptors," Multimedia Tools and Applications, vol. 53, no. 1, pp. 119150, 2011.

5. A. Capar, B. Kurt, and M. Gokmen, "Gradient-based shape descriptors," Machine Vision and Applications, vol. 20, no. 6, pp. 365-378, 2009.

6. C. M. Pun and C. F. Wong, "Fast and robust color feature extraction for content-based image retrieval," International Journal of Advancements in Computing Technology, vol. 3, no. 6, pp. 75-83, 2011.

7. M. B. Rao, B. P. Rao, and A. Govardhan, "Content based image retrieval using dominant color, texture and shape," International Journal on Engineering Science and Technology, vol. 3, no. 4, pp. 2887-2896, 2011.

8. J. Yue, Z. Li, L. Liu, and Z. Fu, "Content-based image retrieval using color and texture fused features," Mathematical and Computer Modelling, vol. 54, no. 3-4, pp. 11211127, 2011.

9. C. Kavitha, B. P. Rao, and A. Govardhan, "Image retrieval based on combined features of image sub-blocks," International Journal on Computer Science and Engineering, vol. 3, no. 4, pp. 1429-1438, 2011.

10. W. H. Yeh and Y. I. Chang, "An efficient iconic indexing strategy for image rotation and reflection in image databases," Journal of Systems and Software, vol. 87, no. 7, pp. 1184-1195, 2008.

11. S. D. Newsam and C. Kamath, "Retrieval using texture features in high resolution multi-spectral satellite imagery," in Data Mining and Knowledge Discovery: Theory, Tools, and 
Technology VI (Proceedings of SPIE), Bellingham, WA: SPIE, pp. 21-32, 2004

12. Y. Li and T. Bretschneider, "Supervised content-based satellite image retrieval using piecewise defined signature similarities," in Proceedings of the IEEE International Geoscience and Remote Sensing Symposium, Toulouse, France, 2003, pp. 734-736.

13. L. Niu, L. Ni, W. Lu, and M. Yuan, "A method of remote sensing image retrieval based on ROI," in Proceeding of the 3rd International Conference on Information Technology and Applications, Sydney, Australia, 2005, pp. 226-229.

14. N. Sawant, S. Chandran, and B. Krishna Mohan, "Retrieving images for remote sensing applications," in Proceedings of the 5th Indian Conference on Computer Vision, Graphics and Image Processing, Madurai, India, 2006, pp. 849-860.

15. A. P. Wang and S. G. Wang, "Content-based high-resolution remote sensing image retrieval with local binary patterns," in Geoinformatics 2006: Remotely Sensed Data and Information (Proceedings of SPIE), Bellingham, WA: SPIE, 2006.

16. H. Shahbazi, P. Kabiri, and M. Soryani, "Content based multispectral image retrieval using independent component analysis," in Proceedings of the 1st International Congress on Image and Signal Processing, Sanya, China, 2008, pp. 485489.

17. D. Peijun, C. Yunhao, T. Hong, and F. Tao, "Study on content-based remote sensing image retrieval," in Proceedings of the IEEE International Geoscience and Remote Sensing Symposium, Seoul, Korea, 2005.

18. P. Maheshwary and N. Srivastava, "Retrieval of remote sensing images using color, texture, and spectral feature," International Journal of Engineering Science and Technology, vol. 2, no. 9, pp. 4306-4311, 2010.

19. S. Ait-Aoudia, R. Mahiou, and B. Benzaid, "YACBIR: yet another content based image retrieval system," in Proceedings of the14th International Conference on Information Visualisation, London, UK, 2010, pp. 570-575.

20. N. Ruan, N. Huang, and W. Hong, "Semantic-based image retrieval in remote sensing archive: an ontology approach," in Proceedings of the IEEE International Conference on Geoscience and Remote Sensing Symposium, Denver, CO, 2006, pp. 2903-2906.

21. L. Wei, W. Weihong, and L. Feng, "Research on remote sensing image retrieval based on geographical and semantic features," in Proceedings of the International Conference on image Analysis and Signal Processing, Taizhou, China, 2009, pp. 162-165.

22. E. El-Qawasmeh, "A quadtree-based representation technique for indexing and retrieval of image databases," Journal of Visual Communication and Image Representation, vol. 14, no. 3, pp. 340-357, 2003.

23. Q. Wan, M. Wang, X. Zhang, S. Jiang, and Y. Xie, "High resolution remote sensing image retrieval using quin-tree and multi-feature histogram," Journal of Geo-Information Science, vol. 12, no. 2, pp. 275-280, 2010.
24. S. Y. Lee and F. J. Hsu, "2D C-string: a new spatial knowledge representation for image database systems," Pattern Recognition, vol. 23, no. 10, pp. 1077-1087, 1990.

25. P. W. Huang, L. Hsu, Y. W. Su, and P. L. Lin, "Spatial inference and similarity retrieval of an intelligent image database system based on object's spanning representation," Journal of Visual Languages and Computing, vol. 19, no. 6, pp. 637651, 2008.

26. P. W. Huang and C. H. Lee, "Image database design based on 9D-SPA representation for spatial relations," IEEE Transactions on Knowledge and Data Engineering, vol. 16, no. 12, pp. 1486-1496, 2004.

27. A. J. T. Lee, R. W. Hong, W. M. Ko, W. K. Tsao, and H. H. Lin, "Mining spatial association rules in image databases," Information Sciences, vol. 177, no. 7, pp. 1593-1608, 2007.

28. C. Urdiales, M. Dominguez, C. de Trazegnies, and F. Sandoval, "A new pyramid-based color image representation for visual localization," Image and Vision Computing, vol. 28, no. 1, pp. 78-91, 2010.

29. N. M. Khan and I. S. Ahmad, "An efficient signature representation for retrieval of spatially similar images," Signal, Image and Video Processing, vol. 6, no. 1, pp. 55-70, 2012.

30. Q. Tan, Z. Liu, and W. Shen, "An algorithm for object-oriented multi-scale remote sensing image segmentation," Journal of Beijing Jiaotong University, vol. 31, no. 4, pp. 111119, 2007.

31. K. L. Tan, B. C. Ooi, and L. F. Thiang, "Indexing shapes in image databases using the centroid-radii model," Data Knowledge Engineering, vol. 32, no. 3, pp. 271-289, 2000.

32. D. Cantone, A. Ferro, A. Pulvirenti, D. R. Recupero, and D. Shasha, "Antipole tree indexing to support range search and k-nearest neighbor search in metric spaces," IEEE Transactions on Knowledge and Data Engineering, vol. 17, no. 4, pp. 535-550, 2005.

33. A. Vetro, "MPEG-7 applications document v.10," ISO/IEC JTC1/SC29/WG11/N3934, 2001.

34. S. E. Grigorescu, N. Petkov, and P. Kruizinga, "Comparison of texture features based on Gabor filters," IEEE Transactions on Image Processing, vol. 11, no. 10, pp. 1160-1167, 2002.

35. C. Nastar, M. Mitschke, and C. Meilhac, "Efficient query refinement for image retrieval," in Proceedings of the IEEE Computer Society Conference on Computer Vision and Pattern Recognition, Santa Barbara, CA, 1998, pp. 547-552.

36. T. Liu, Q. Du, and H. Yan, "Spatial similarity assessment of point cluster," Geomatics and Information Science of Wuhan University, vol. 36, no. 10, pp. 1149-1152, 2011.

37. P. Kruizinga, N. Petkov, and S. E. Grigorescu, "Comparison of texture features based on Gabor filters," in Proceedings of the 10th International Conference on Image Analysis and Processing, Venice, Italy, 1999, pp. 142-147.

38. G. Salton and M. J. McGill, Introduction to Modern Information Retrieval, New York, NY: McGraw-Hill, 1983. 


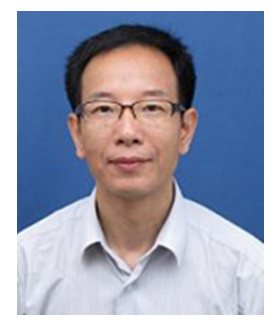

\section{Zhi Zeng}

Zhi Zeng is currently a teacher in the Department of Computer Science at Huizhou University in Guangdong Province, China. He received his B.E. in Computer Science from Jiangxi Normal University, M.E. in Computer Science from Zhejiang University, and Ph.D. in GIS from Zhejiang University, China. His research interests include GIS and remote sensing image processing with a focus on massive data processing in grid computing and cloud computing. In recent years, he has published more than 10 papers.

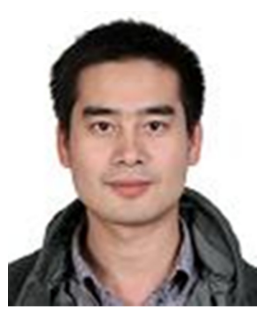

\section{Zhenhong Du}

Zhenhong $\mathrm{Du}$ is an associate professor in the Department of Earth Science at Zhejiang University. He received both his M.S. and Ph.D. in the field of GIS from Zhejiang University, China. He also completed postdoctoral research in GIS at Zhejiang University. His research interest areas include information development in marine remote sensing, massive data processing in grid computing or cloud computing, three-dimensional city, underground pipeline, water conservancy, etc. In recent years, he has published more than 20 papers.

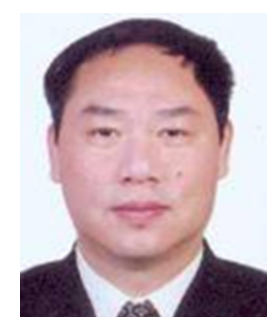

\section{Renyi Liu}

Renyi Liu is a professor in the Department of Earth Science at Zhejiang University, and the director of the Institute of Geographical Information Science. He received his B.S. in Geomatics and Geodesy from Wuhan University, and his Ph.D. in the field of Geographical Information Science from Zhejiang University, China. His research interest areas include information development in marine remote sensing, basic geography information, three-dimensional city, underground pipeline, water conservancy, etc. In recent years, he has published more than 30 papers. 